\title{
Nonsteady dynamics properties of a domain wall for the creep state under an alternating driving field
}

\author{
N.J. Zhou ${ }^{1 *}$, and B. Zheng ${ }^{2}$ \\ ${ }^{1}$ Department of Physics, Hangzhou Normal University, Hangzhou 310036, P.R. China \\ ${ }^{2}$ Department of Physics, Zhejiang University, Hangzhou 310027, P.R. China
}

\begin{abstract}
With Monte Carlo simulations, the nonsteady dynamics properties of a domain wall have been systematically investigated for the thermally activated creep state under an alternating driving field. Taking the driven random-field Ising model in two dimensions as an example, two distinct growth stages of the domain interface are identified with both the correlation length and roughness function. One stage belongs to the universality class of the random depositions, and the other to that of the quenched Edwards-Wilkinson equation. In the latter case, due to the dynamic effect of overhangs, the domain interface may exhibit an intrinsic anomalous scaling behavior, different from that of the quenched Edwards-Wilkinson equation.

PACS numbers: 64.60.Ht, 05.10.Ln, 75.60.Ch
\end{abstract}

* corresponding author; email: zhounengji@hznu.edu.cn 


\section{INTRODUCTION}

In recent years, the domain-wall motion has become a source of much experimental and theoretical research [1 8]. The dynamics under an alternating driving field has attracted extensive interests in vortex lattices [9, 10], liquid crystals [11], ferromagnetic/ferroelectric materials [12 14] and crystalline solids [15]. In particular, the magnetic domain-wall dynamics is an important topic in nanomaterials, thin films and semiconductors, because of its potential technological applications including magnetic random access memories and logic devices [16 18]. In the experiments of ultrathin ferromagnetic and ferroelectric films, considerable attention is devoted to the complex susceptibility $\left(\chi=\chi^{\prime}-i \chi^{\prime \prime}\right)$ [19 21], which depicts the domain-wall motion. Four dynamic states are observed in the Cole-Cole diagram of $\chi^{\prime}$ vs. $\chi^{\prime \prime}$, which are relaxation, creep, sliding and switching. Recently, experimental evidences of the relaxation-to-creep dynamic transition have been found, not only in ultrathin ferromagnetic trilayers and ferroelectric films $\mid 20-23]$, but also in liquid crystals, ferroelastic materials and molecular ferrimagnets [11, 24, 25]. However, theoretical understanding of the transition is limited, especially for the growth process of the correlation length [26, 27].

For the creep state at low temperatures and low frequencies, one observes an inverse power-law behavior for the complex susceptibility $\chi(f)=\chi_{\infty}\left[1+(i 2 \pi f \tau)^{-\beta}\right][2]$. Here $\chi_{\infty}$ denotes the bulk background susceptibility when the frequency $f \rightarrow \infty, \tau$ is the characteristic relaxation time, and $\beta$ is the creep exponent. According to scaling arguments, a theoretical value $\beta=(2-2 \zeta) / z \approx 0.5$ is expected with the roughness exponent $\zeta \approx 2 / 3$ and dynamic exponent $z \approx 1.33$ [21]. While experimental values of $\beta$ vary from 0.2 to 0.65 in ultrathin ferromagnetic and ferroelectric films [20-22]. Hence, it remains much challenging to understand the creep exponent.

Up to date, theoretical tools for describing the domain-wall motion are typically based on the Edwards-Wilkinson equation with quenched disorder (QEW) [6, 28 32]. With this equation, the dynamics properties for the creep state under a constant driving field or zero field have been well understood [33 35]. It can be viewed as a thermally activated hopping movement from one local energy minimum to the next, dominated by the energy barrier $U_{B}$ that must be overcome. The energy barrier grows as a power law $U_{B}(\xi) \sim \xi^{\psi}$, responsible for the logarithmical growth of the correlation length $\xi(t) \sim(\ln t)^{1 / \psi}[36$, , 37]. An effective energy barrier exponent $\psi \approx 0.49$ has been numerically measured [38, 39], and the roughness 
exponent $\zeta \approx 2 / 3$ has also been estimated from the kinetic roughening of the domain wall [33, 34]. However, few works deal with the creep dynamics under an alternating driving field. Moreover, detailed microscopic structures and interactions of real materials are not concerned in the phenomenological QEW equation [28].

To further understand the creep dynamics from a more fundamental viewpoint, we should build lattice models which allow a closer comparison between theory and experiment. The driven random-field Ising model (DRFIM) is a candidate, which has been used to understand the dynamic transitions in ferroic systems [40-42]. Despite not including all interactions in real materials, it at least captures robust features of the domain-wall motion. Very recently, the creep motion of a domain wall driven by a constant field has been numerically investigated with the DRFIM model, and the results are comparable with experiments [43].

Taking the two-dimensional (2D) DRFIM model as an example, we conduct a comprehensive study on the creep dynamics under an alternating driving field. With Monte Carlo simulations, we accurately determine the scaling exponents $\beta, \psi$ and $\zeta$, and identify the universality classes, in comparison with those of the QEW equation and experiments. In Sec. II, the model and scaling analysis are described, and in Sec. III, the numerical results are presented. Finally, Sec. IV includes the conclusions.

\section{MODEL AND SCALING ANALYSIS}

The DRFIM model is defined by the following Hamiltonian

$$
\mathcal{H}=-J \sum_{\langle i j\rangle} S_{i} S_{j}-\sum_{i}\left[h_{i}+H(t)\right] S_{i}
$$

where $S_{i}= \pm 1$ is a Ising spin at site $i$ of the lattice, $\langle i j\rangle$ denotes a nearest-neighbor pair of spins, and $h_{i}$ is a quenched random field uniformly distributed within an interval $[-\Delta, \Delta]$. We use a homogeneous alternating driving field $H(t)=H_{0} \cos (2 \pi f t)$, and set the coupling

constant $J=1$ [42]. In order to make sure that the dynamic evolution of spins occurs at or around the domain wall, we restrict the temperature $T \leq 0.66$, the disorder strength $\Delta \leq 2.0$ and the driving field $H_{0} \leq 0.5$. Simulations are performed on a rectangular lattice $L_{x} \times L_{y}$ with the antiperiodic and periodic boundary conditions along the $x$ and $y$ directions, respectively.

The initial state is a semiordered state with a perfect domain wall in the $y$ direction. To 
eliminate the pinning effect irrelevant for the disorder, we rotate the lattice such that the initial domain wall orients in the (11) direction [41, 44]. After preparing the initial state, we update spins with the heat-bath algorithm [45]. As time evolves, the domain wall moves and roughens, while the bulk remains unchanged. Therefore, the domain wall can also be called a domain interface [46, 47]. Main results of numerical simulations are presented with the lattice size $L_{x}=25$ and $L_{y}=512$, up to $t_{\max }=400000$ Monte Carlo steps (MCS). Here MCS is defined by $L_{x} \times L_{y}$ single-spin-flips attempts. For each set of model parameters $\left(T, \Delta, H_{0}, f\right)$, more than 10000 samples are performed for average. Errors are estimated by dividing the samples into three subgroups. If the fluctuation of the curve in the time direction is comparable with or larger than the statistical error, it will also be taken into account. Additional simulations with $L_{x}=50$ are performed to confirm that the finite-size effect is negligible.

Denoting a spin at site $(x, y)$ by $S_{x y}(t)$, we first introduce the height function

$$
h(y, t)=\sum_{x=1}^{L_{x}} S_{x y}(t),
$$

and then define the position of the domain interface

$$
h(t)=\frac{1}{2}\left[\langle h(y, t)\rangle+L_{x}\right]
$$

Here $\langle\cdots\rangle$ represents not only the statistic average over Monte Carlo samples, but also the average in the $y$ direction. After the stationary magnetic hysteresis loop is obtained at $t>t_{0}$, the complex susceptibility can be calculated by [28, 42],

$$
\chi(f, T)=\frac{1}{P H_{0}} \int_{0}^{P} h(t) e^{-i 2 \pi f t} d t,
$$

where $P=1 / f$ is the time period of the alternating driving field.

With the height function $h(y, t)$ at hand, the roughness function $\omega^{2}(t)$ and the correlation function $C(r, t)$ are defined respectively by

$$
\omega^{2}(t)=\left\langle h(y, t)^{2}\right\rangle-\langle h(y, t)\rangle^{2}
$$

and

$$
C(r, t)=\langle h(y, t) h(y+r, t)\rangle-\langle h(y, t)\rangle^{2} .
$$


$\omega^{2}(t)$ and $C(r, t)$ describe the roughening of the domain interface in the $x$ direction and the growth of the spatial correlation in the $y$ direction, respectively. To reveal the characteristics of the thermally activated creep dynamics, we introduce the creep susceptibility

$$
D \chi^{\prime}(f, T)=\chi^{\prime}(f, T)-\chi^{\prime}(f, T=0),
$$

and the pure roughness function

$$
D \omega^{2}(t)=\omega^{2}(t)-\omega^{2}(t, T=0) .
$$

To detect overhangs generated in the creep motion, another two definitions of the height functions, $h^{+}(t)$ and $h^{-}(t)$, are introduced by the envelopes of the positive and negative spins, respectively [44]. It is believed that the difference $D h(t)=h^{+}(t)-h^{-}(t)$ describes the average size of overhangs.

According to the phenomenological scaling arguments [48], a power-law dispersion of the creep susceptibility is obtained for the creep dynamics under an alternating driving field,

$$
D \chi^{\prime}(f) \sim(1 / f)^{\beta} .
$$

For the $\xi$-length domain-wall segments, a certain hopping time $t \sim \exp \left(U_{B}(\xi) / T\right)$ is required to overcome the energy barrier $U_{B}(\xi)$ [38]. Assuming that the energy barrier scales as $U_{B}(\xi) \sim \xi^{\psi}$, one may deduce

$$
D \xi(t) \sim[T \ln (t)]^{1 / \psi} .
$$

Here $D \xi(t)=\xi(t)-\xi(t, T=0)$ is the so-called creep correlation length, and $\psi$ is the energy barrier exponent.

For a sufficiently large lattice $L \gg \xi(t)$, the dynamic behavior of $\xi(t)$ can be extracted from the correlation function [29, 49],

$$
C(r, t)=\omega^{2}(t) \widetilde{C}(r / \xi(t)),
$$

where $\widetilde{C}(s)$ is the scaling function with $s=r / \xi(t)$, and $\omega^{2}(t)$ is the roughness function defined in Eq. (5). In the kinetic roughening of the domain interface, a power-law scaling behavior of the pure roughness function is expected with the roughness exponent $\zeta$,

$$
D \omega^{2}(t) \sim[D \xi(t)]^{2 \zeta} .
$$

Meanwhile, one may determine the local roughness exponent $\zeta_{l o c}$ by fitting $C(r, t)$ with an empirical scaling form [50],

$$
C(r, t)=A[\tanh (r / \xi(t))]^{2 \zeta_{l o c}} .
$$




\section{MONTE CARLO SIMULATIONS}

\section{A. Numerical results}

In Fig. 1, the spectrum of the creep susceptibility $D \chi^{\prime}(f)$ defined in Eq. (7) is plotted on a $\log$ - $\log$ scale at the temperature $T=0.33$, the strength of the disorder $\Delta=1.5$ and the driving field $H_{0}=0.01$. To obtain stationary results, the data in a waiting time $t_{0}=20$ periods are skipped in the computation of $\chi(f, T)$. A power-law behavior is observed but with certain corrections to scaling. A direct measurement from the slope yields the exponent $\beta=0.55(2)$, and the correction in the form $y=a x^{\beta}(1-c / x)$ extends the fitting to the early times but with the same value of $\beta$ within the error bar. For comparison, the creep exponents at other temperatures $T=0.025,0.05,0.1,0.2$ and 0.66 are measured. As shown in the inset, the result $\beta \approx 0.2$ jumps suddenly to 0.5 around $T=0.33$. In order to understand the underlying dynamic mechanism, we investigate the nonsteady dynamics in the following.

Taking the set of model parameters $\left(T=0.33, \Delta=1.5, H_{0}=0.01\right.$ and $\left.f=10^{-4} \mathrm{~Hz}\right)$ as an example, the correlation function $C(r, t)$ is displayed as a function of $r$ in Fig. 2(a). According to Eq. (13), a perfect fitting to the numerical data is observed, and the local roughness exponent $\zeta_{l o c}=0.65(1)$ is measured. Based on the scaling form of $C(r, t)$ in Eq. (11), numerical data of different time $t$ nicely collapse to the curve at $t^{\prime}=400000 \mathrm{MCS}$ by rescaling $r$ to $\left[\xi\left(t^{\prime}\right) / \xi(t)\right] r$ and $C(r, t)$ to $\left[\omega^{2}\left(t^{\prime}\right) / \omega^{2}(t)\right] C(r, t)$. With the data-collapse technique [43], we extract the nonequilibrium correlation length $\xi(t)$ from the correlation function $C(r, t)$. In the inset, the dynamic evolution of $\xi(t)$ is displayed on a log-log scale. The significant deviation from the power-law behavior indicates that the correlation length $\xi(t)$ does not obey the usual growth law $\xi(t) \sim t^{1 / z}[38$, 51]. Additionally, a time-independent correlation length $\xi=L_{c}$ is observed at $T=0$ for the relaxation state, irrelevant to the creep dynamics. Therefore, we define the creep correlation length $D \xi(t)$ by subtracting the relaxation length $L_{c}$, and introduce a dimensionless correlation length $D \xi(t) / L_{c}$.

In Fig. 2(b), the creep correlation length $D \xi(t) / L_{c}$ is displayed as a function of $\ln t$ at different $T$ on a log-log scale. Power-law behaviors are observed, and the effective energy barrier exponents $1 / \psi=0.98(1), 0.98(1), 1.16(1), 1.73(2), 1.84(2)$ and $1.75(3)$ are estimated from the slopes of the curves for $T=0.025,0.05,0.1,0.2,0.33$ and 0.66 , respectively. To confirm the scaling form in Eq. (10), $D \xi(t) / L_{c}$ vs. $T \ln t$ is plotted in Fig. 3(a) on a double- 
log scale. Data of different $T$ nicely collapse to a master curve, and two distinct scaling regimes are detected with the slopes $1 / \psi=1.00(1)$ and $1.80(2)$. Between the two regimes, a dynamic crossover occurs at $T \ln t \approx 1$ and $D \xi(t) \approx 0.5 L_{c}$. Then we extract the characteristic of the energy barrier

$$
U_{B} \sim T \ln t \sim\left\{\begin{array}{ll}
2 D \xi(t) / L_{c}, & D \xi(t) \ll 0.5 L_{c} \\
1.5\left(D \xi(t) / L_{c}\right)^{0.56}, & D \xi(t) \gg 0.5 L_{c}
\end{array},\right.
$$

in the small- $D \xi(t)$ and large- $D \xi(t)$ scaling regimes, respectively.

With the creep correlation length $D \xi(t) / L_{c}$ at hand, we measure the roughness exponent $\zeta$ in Eq. (12). Since the amplitude of the alternating field $H_{0}=0.01$ is much smaller than the depinning field $H_{c}=1.2933(2)$ [41], the roughness exponent is actually the equilibrium exponent, though the equilibrium state is not yet reached [38]. In Fig. 33(b), $D \omega^{2}(t)$ is plotted as a function of $D \xi(t) / L_{c}$ on a log-log scale. Similarly, data collapse of different $T$ is displayed with different symbols. In the small- $D \xi(t)$ regime, the slope of the master curve yields the roughness exponent $\zeta=0.53(1)$, close to $1 / 2$. It suggests that the domain interface belongs to the universality class of the random depositions [52 55]. In the large$D \xi(t)$ regime, $\zeta=0.68(1)$ is estimated, in good agreement with the equilibrium value $\zeta_{e q}=2 / 3$ of the QEW equation [34, 36, 38, 56]. Hence, it belongs to the universality class of the QEW equation. In the inset, however, a noticeable increase of $\zeta$ is observed at the high temperature $T=0.66$, and the asymptotic value is $\zeta=1.00(2)$.

To understand the unexpectedly large roughness exponent at $T=0.66$, we examine the existence of overhangs in the creep motion [41, 44]. As shown in Fig. 4(a), black and red lines represent the time evolutions of the height functions $h^{+}(t)$ and $h^{-}(t)$, respectively. The coincidence and noncoincidence of the curves in the upper and lower panels suggest that the contribution of overhangs is negligible at $T=0.2$ and important at $T=0.66$. Besides, the snapshots of the domain walls at the time $t=4 \times 10^{5}$ MCS are also shown in the insets. Overhangs can be observed directly in the lower panel but not in the upper panel. Consequently, it is convincing that the overhangs affect the dynamic evolution of the spin configuration and play an essential role in the increase of the roughness exponent.

Due to the existence of overhangs, the position of the domain interface $h(y, t)$ is not single-valued and the definition of the height function is not unique. As shown in Fig. 4(b), $D \omega^{2}(t)$ and $D \xi(t) / L_{c}$ at $T=0.66$ are displayed for the domain interface $h^{-}(t)$ defined with the envelop of the negative spins, in comparison with those for the domain interface 
$h(t)$ defined with the magnetization in Eq. (3). The roughness exponent $\zeta=0.97(2)$ and the energy barrier exponent $1 / \psi=1.81(4)$ are measured, consistent with $\zeta=1.00(2)$ and $1 / \psi=1.75(3)$ for the domain interface defined with the magnetization. These results again support that the definition of the height function with the magnetization is reasonable.

Besides the temperature, the effects of the quenched disorder and driving field are also investigated. In Fig. 5 (a), the creep correlation length $D \xi(t) / L_{c}$ is displayed as a function of $\Delta^{-\delta} \ln t$ at $T=0.33$ on a log-log scale. Taking $\delta=0.58(1)$ as input, data collapse of different disorders $\Delta=0.5,0.7,1.0$ and 1.5 is demonstrated, and $1 / \psi=1.85(2)$ is determined, close to $1.80(2)$ in Fig. $3($ a). According to the scaling relation $\delta \approx \psi \approx 0.56$, one may derive the scaling form of $U_{B}$ in the large- $D \xi(t)$ regime,

$$
U_{B} \sim T \ln t \sim(D \xi(t) \Delta)^{\psi}
$$

In the inset, the scaling function $\Delta^{-\varepsilon} D \omega^{2}(t)$ vs. $D \xi(t) / L_{c}$ is plotted on a log-log scale. Data of different $\Delta$ nicely collapse together with the parameter $\varepsilon=0.22(1)$ as input. Similarly, the abnormal increase of the roughness exponent from $\zeta=0.68(1)$ to $0.78(1)$ is also induced by the dynamic effect of overhangs.

In Fig. 5(b), the creep dynamics of the domain wall for different frequencies $f$ is presented at the driving field $H_{0}=0.1$ on a $\log$ - $\log$ scale. If the frequency is sufficiently low, e.g., $f=10^{-4} \mathrm{~Hz}$, a power-law behavior of $\xi(t)$ can be observed with the exponent $1 / \psi=1.90(2)$, somewhat larger than the one $1.80(2)$ at $H_{0}=0.01$. Additional simulations at $H_{0}=0.05,0.2$ and 0.5 show that the energy barrier exponent $\psi$ is $H_{0}$-dependent, and data of different $H_{0}$ are unlikely to collapse together. For a high frequency, e.g., $f=1 \mathrm{~Hz}$ corresponding to the

relaxation state [42], $D \xi(t)$ drops obviously at the tail of the curve. It suggests that the nonsteady dynamics properties of the relaxation state are different from those of the creep state.

\section{B. Discussion}

The measurements of scaling exponents at different $T$ are summarized for $H_{0}=0.01$ and $\Delta=1.5$ in Table. II As $T$ increases, the creep exponent $\beta$ changes from $0.23(1)$ to $0.55(2)$, compatible with experimental results in the ferromagnetic and ferroelectric films [22], e.g., $\beta=0.6(1)$ in the ultrathin $\mathrm{Pt} / \mathrm{Co}(0.5 \mathrm{~nm}) / \mathrm{Pt}$ trilayer and $\beta=0.35(2)$ in the periodically 
poled $\mathrm{KTiOPO}_{4}$ [20, 21]. The exponent $\beta=0.52(2)$ measured at the highest temperature $T=0.66$ is consistent with the prediction of the scaling relation $\beta=(2-2 \zeta) / z \approx 0.5$ [21]. According to the general scaling arguments [57], we propose the complex susceptibility $D \chi(f) \sim \ln (1 / f)^{\zeta / \psi} \sim(1 / f)^{k \zeta / \psi}$ with an effective coefficient $k$. Then a novel scaling relation $\beta=k \zeta / \psi$ is obtained. As shown in Table. I, $k \approx \beta \psi / \zeta_{l o c}=0.45(3)$ holds for the whole temperature range. With this scaling relation, one can predict the creep exponent $\beta$ by only measuring $\psi$ and $\zeta$ from the nonsteady dynamics.

Two distinct growth stages of the creep correlation length $D \xi(t)$ are found with the scaling exponents $1 / \psi=1.00(1), \zeta=0.53(1)$ in the small- $D \xi(t)$ scaling regime and $1 / \psi=$ $1.80(2), \zeta=0.68(1)$ in the large- $D \xi(t)$ scaling regime. The results indicate that the former belongs to the universality class of the random depositions, while the later belongs to the universality class of the QEW equation. The two universality classes are separated by the so-called Larkin length $L_{p}$ at which the effects of the quenched disorder and domain-wall elasticity are of the same order [12, 48, 58, 59]. According to Eq. (14), $L_{p} \approx 0.5 L_{c}$ is estimated for the creep dynamics. Now let us recall the growth process of the creep correlation length. At the beginning, the elasticity is dominant. The kinetic roughening of the domain interface is then dominated by thermal fluctuations with $\zeta_{T}=1 / 2$ [30, 33], and the energy barrier is linear with $D \xi(t)$. After $D \xi(t)$ reaches $L_{p}$, the quenched disorder overcomes the elasticity. Then the domain-wall motion can be described by the QEW equation with the nontrivial exponents $\psi=1 / 2$ and $\zeta_{\text {eq }}=2 / 3[38,56]$.

In Table. II, the effects of $\Delta, H_{0}$ and $f$ are uncovered in the large- $D \xi(t)$ regime with the fixed sets of model parameters $\left(T=0.33, H_{0}=0.01, f=10^{-4} \mathrm{~Hz}\right),(T=0.33, \Delta=1.5, f=$ $\left.10^{-4} \mathrm{~Hz}\right)$ and $\left(T=0.33, \Delta=1.5, H_{0}=0.1\right)$, respectively. For a moderate disorder, i.e., $0.5 \leq \Delta \leq 1.5$, a robust value $\psi=0.54(1)$ is determined, close to $\psi=1 / 2$ of the $\mathrm{QEW}$ equation. According to Eq. (15), the hopping time $t \sim \exp \left(\left[D \xi / L_{p}\right]^{\psi} / T\right)$ is derived with $L_{p} \sim 1 / \Delta$ [12, 36], consistent with the ones in Refs. [38, 60]. The factor $\Delta^{\psi} / T$ shows that the hopping process is determined by the competition between the quenched disorder and thermal noise [33]. Since $1 / \psi$ increases monotonically with $H_{0}$ and the curve $D \xi(t)$ drops at the tail, further studies are needed to derive the exact functional form on $H_{0}$ and $f$.

For the kinetic roughening of the domain interface, a robust value $\zeta=0.68(1)$ is determined in the large- $D \xi(t)$ scaling regime at different $T, \Delta, H_{0}$ and $f$. The scaling relation $\zeta=\zeta_{l o c}<1$ indicates that the domain interfaces belongs to the Family-Vicsek universality 
class [61]. When $D \xi(t)$ exceeds a certain threshold, however, $\zeta$ differs from $\zeta_{\text {loc }}$ by more than 15 percent not only at a higher temperature $T=0.66$, but also at weaker quenched disorders $\Delta=0.1,0.5$, stronger driving fields $H_{0}=0.2,0.5$ and higher frequencies $f=10^{-1} \mathrm{~Hz}, 1 \mathrm{~Hz}$. A similar phenomenon has also been observed in Ref. [34] where a crossover of the roughness exponent from $\zeta_{l o c}=2 / 3$ to $\zeta=1.25$ is obtained for different driving fields. It suggests that in this case the domain interface is no longer single-valued and one-dimensional. As a consequence, the domain interface belongs to a new universality class with intrinsic anomalous scaling and spatial multiscaling [41, 61].

\section{CONCLUSION}

With Monte Carlo simulations, we have explored the nonsteady dynamics properties of a domain wall for the creep state under an alternating driving field. Since the phenomenological QEW equation contains little microscopic information, lattice models based on microscopic structures and interactions are considered. Taking the 2D DRFIM model as an example, two distinct growth stages of the domain interface are identified with both the creep correlation length $D \xi(t)$ and the pure roughness function $D \omega^{2}(t)$. The small- $D \xi(t)$ regime corresponds to the universality class of the random depositions with the scaling exponents $\psi=1$ and $\zeta_{T}=1 / 2$, while the large- $D \xi(t)$ one belongs to the universality class of the QEW equation with $\psi=1 / 2$ and $\zeta_{\text {eq }}=2 / 3$.

However, due to the dynamic effect of overhangs, the roughness exponent $\zeta$ may significantly deviate from 2/3 of the QEW equation at either higher temperatures, weaker quenched disorders, stronger driving fields or higher frequencies, but comparable with experiments [62, 63]. The result $\zeta>\zeta_{l o c}=2 / 3$ indicates that the domain interface belongs to a new universality class with intrinsic anomalous scaling and spatial multiscaling.

In addition, as the temperature increases, the creep exponent $\beta$ measured from the stationary magnetic hysteresis loops changes from $0.23(1)$ to $0.55(2)$, compatible with the experimental measurements [20-22]. The temperature-independent scaling relation $\beta=k \zeta / \psi$ is then observed with the coefficient $k=0.45(3)$. With this scaling relation, one can predict the creep exponent $\beta$ by only measuring $\psi$ and $\zeta$ from the nonsteady dynamics.

Acknowledgements: This work was supported in part by the National Natural Science Foundation of China (under Grant Nos. 11205043, 11375149 and 11304072), the Zhejiang 
Provincial Natural Science Foundation (under Grants No. LQ12A05002), and the funds from Hangzhou City for supporting Hangzhou-City Quantum Information and Quantum Optics Innovation Research Team.

[1] G. Tatara and H. Kohno, Phys. Rev. Lett. 92, 086601 (2004).

[2] W. Kleemann, Annu. Rev. Mater. Res. 37, 415 (2007).

[3] E. Martinez, L. L. Diaz, L. Torres, C. Tristan, and O. Alejos, Phys. Rev. B 75, 174409 (2007).

[4] P. J. Metaxas, J. P. Jamet, A. Mougin, M. Cormier, J. Ferré, V. Baltz, B. Rodmacq, B. Dieny, and R. L. Stamps, Phys. Rev. Lett. 99, 217208 (2007).

[5] K. J. Kim, J. C. Lee, S. M. Ahn, K. S. Lee, C. W. Lee, Y. J. Cho, S. Seo, K. H. Shin, S. B. Choe, and H. W. Lee, Nature 458, 740 (2009).

[6] F. Schütze and T. Nattermann, Phys. Rev. B 83, 024412 (2011).

[7] I. M. Miron, T. Moore, H. Szambolics, L. D. B. Prejbeanu, S. Auffret, B. Rodmacq, S. Pizzini, J. Vogel, M. Bonfim, A. Schuhl, and G. Gaudin, Nature Mater. 10, 419 (2011).

[8] J. C. Lee, K. J. Kim, J. Ryu, K. W. Moon, S. J. Yun, G. H. Gim, K. S. Lee, K. H. Shin, H. W. Lee, and S. B. Choe, Phys. Rev. Lett. 107, 067201 (2011).

[9] M. I. Dolz, A. B. Kolton, and H. Pastoriza, Phys. Rev. B 81, 092502 (2010).

[10] W. P. Cao, M. B. Luo, and X. Hu, New J. Phys. 14, 013006 (2012).

[11] W. Jeżewski, W. Kuczyński, and J. Hoffmann, Phys. Rev. B 77, 094101 (2008).

[12] F. Kagawa, Y. Onose, Y. Kaneko, and Y. Tokura, Phys. Rev. B 83, 054413 (2011).

[13] N. J. Steinke, T. A. Moore, R. Mansell, J. A. C. Bland, and C. H. W. Barnes, Phys. Rev. B 86, $184434(2012)$.

[14] K. Zhernenkov, D. Gorkov, B. P. Toperverg, and H. Zabel, Phys. Rev. B 88, 020401(R) (2013).

[15] L. Laurson and M. J. Alava, Phys. Rev. Lett. 109, 155504 (2012).

[16] M. Yamanouchi, J. Ieda, F. Matsukura, S. E. Barnes, S. Maekawa, and H. Ohno, Science 317, 1726 (2007).

[17] M. Hayashi, L. Thomas, R. Moriya, C. Rettner, and S. S. P. Parkin, Science 320, 209 (2008).

[18] S. S. P. Parkin, M. Hayashi, and L. Thomas, Science 320, 190 (2008).

[19] A. Venimadhav, D. Chandrasekar, and J. K. Murthy, Appl. Nanosci. 3, 25 (2013). 
[20] T. Braun, W. Kleemann, J. Dec, and P. A. Thomas, Phys. Rev. Lett. 94, 117601 (2005).

[21] W. Kleemann, J. Rhensius, O. Petracic, J. Ferré, J. P. Jamet, and H. Bernas, Phys. Rev. Lett. 99, 097203 (2007).

[22] W. Kleemann, J. Dec, S. A. Prosandeev, T. Braun, and P. A. Thomas, Ferroelectrics 334, 3 (2006).

[23] S. M. Yang, J. Y. Jo, T. H. Kim, J. G. Yoon, T. K. Song, H. N. Lee, Z. Marton, S. Park, Y. Jo, and T. W. Noh, Phys. Rev. B 82, 174125 (2010).

[24] R. J. Harrison, S. A. T. Redfern, and E. K. H. Salje, Phys. Rev. B 69, 144101 (2004).

[25] F. Mushenok, O. Koplak, and R. Morgunov, Eur. Phys. J. B 84, 219 (2011).

[26] T. Nattermann, V. Pokrovsky, and V. M. Vinokur, Phys. Rev. Lett. 87, 197005 (2001).

[27] A. Glatz, T. Nattermann, and V. Pokrovsky, Phys. Rev. Lett. 90, 047201 (2003).

[28] O. Petracic, A. Glatz, and W. Kleemann, Phys. Rev. B 70, 214432 (2004).

[29] A. B. Kolton, A. Rosso, E. V. Albano, and T. Giamarchi, Phys. Rev. B 74, 140201(R) (2006).

[30] O. Duemmer and W. Krauth, Phys. Rev. E 71, 061601 (2005).

[31] E. E. Ferrero, S. Bustingorry, and A. B. Kolton, Phys. Rev. E 87, 032122 (2013).

[32] F. Schütze, Phys. Rev. E 81, 051128 (2010).

[33] A. B. Kolton, A. Rosso, and T. Giamarchi, Phys. Rev. Lett. 94, 047002 (2005).

[34] A. B. Kolton, A. Rosso, T. Giamarchi, and W. Krauth, Phys. Rev. B 79, 184207 (2009).

[35] P. Chauve, T. Giamarchi, and P. L. Doussal, Phys. Rev. B 62, 6241 (2000).

[36] C. Monthus and T. Garel, Phys. Rev. E 78, 041133 (2008).

[37] C. Monthus and T. Garel, J. Stat. Mech. 2008, P07002 (2008).

[38] A. B. Kolton, A. Rosso, and T. Giamarchi, Phys. Rev. Lett. 95, 180604 (2005).

[39] C. Monthus and T. Garel, J. Phys. A: Math. Theor. 41, 115002 (2008).

[40] F. Colaiori, G. Durin, and S. Zapperi, Phys. Rev. Lett. 97, 257203 (2006).

[41] N. J. Zhou, B. Zheng, and Y. Y. He, Phys. Rev. B 80, 134425 (2009).

[42] N. J. Zhou, B. Zheng, and D. P. Landau, Europhys. Lett. 92, 36001 (2010).

[43] R. H. Dong, B. Zheng, and N. J. Zhou, Europhys. Lett. 98, 36002 (2012).

[44] N. J. Zhou and B. Zheng, Phys. Rev. E 82, 031139 (2010).

[45] B. Zheng, Int. J. Mod. Phys. B 12, 1419 (1998).

[46] N.J. Zhou and B. Zheng, Europhys. Lett. 78, 56001 (2007).

[47] N. J. Zhou and B. Zheng, Phys. Rev. E 77, 051104 (2008). 
[48] A. A. Fedorenko and S. Stepanow, Phase Transitions 78, 817 (2005).

[49] N. J. Zhou, B. Zheng, and J. H. Dai, Phys. Rev. E 87, 022113 (2013).

[50] M. Jost and K. D. Usadel, Phys. Rev. B 54, 9314 (1996).

[51] R. H. Dong, B. Zheng, and N. J. Zhou, Europhys. Lett. 99, 56001 (2012).

[52] H. N. Yang and T. M. Lu, Phys. Rev. B 51, 2479 (1995).

[53] S. D. Sarma, C. J. Lanczycki, R. Kotlyar, and S. V. Ghaisas, Phys. Rev. E 53, 359 (1996).

[54] A. A. Fedorenko, V. Mueller, and S. Stepanow, Phys. Rev. B 70, 224104 (2004).

[55] E. Agoritsas, V. Lecomte, and T. Giamarchi, Phys. Rev. E 87, 062405 (2013).

[56] J. L. Iguain, S. Bustingorry, A. B. Kolton, and L. F. Cugliandolo, Phys. Rev. B 80, 094201 (2009).

[57] T. Nattermann, Y. Shapir, and I. Vilfan, Phys. Rev. B 42, 8577 (1990).

[58] A. Tanguy and T. Vettorel, Eur. Phys. J. B 38, 71 (2004).

[59] T. Nogawa, K. Nemoto, and H. Yoshino, Phys. Rev. B 77, 064204 (2008).

[60] F. Corberi, L. F. Cugliandolo, and H. Yoshino, in Dynamical Heterogeneities in Glasses, Colloids, and Granular Media (2011) p. 370.

[61] J. J. Ramasco, J. M. López, and M. A. Rodríguez, Phys. Rev. Lett. 84, 2199 (2000).

[62] S. Lemerle, J. Ferré, C. Chappert, V. Mathet, T. Giamarchi, and P. L. Doussal, Phys. Rev. Lett. 80, 849 (1998).

[63] K. S. Lee, C. W. Lee, Y. J. Cho, S. Seo, D. H. Kim, and S. B. Choe, IEEE. Trans. Magn. 45, 2548 (2009). 
TABLE I: Scaling exponents of the creep dynamics at different temperatures $T$. The scaling relation $\psi \beta / \zeta_{\text {loc }}=0.45(3)$ holds within error bars. The roughness exponents $\zeta=0.53(1)$ and $0.68(1)$ are measured in the small- $D \xi(t)$ regime (central columns) and large- $D \xi(t)$ regime (right columns), respectively, in good agreement with the local ones $\zeta_{l o c}$.

\begin{tabular}{l|cccc|cc}
\hline \hline $\mathrm{T}$ & 0.025 & 0.05 & 0.1 & 0.2 & 0.33 & 0.66 \\
\hline$\beta$ & $0.23(1)$ & $0.23(1)$ & $0.26(1)$ & $0.38(1)$ & $0.55(2)$ & $0.52(2)$ \\
$1 / \psi$ & $0.98(1)$ & $0.98(1)$ & $1.16(1)$ & $1.73(2)$ & $1.84(2)$ & $1.75(3)$ \\
$\zeta_{l o c}$ & $0.50(1)$ & $0.51(1)$ & $0.50(1)$ & $0.51(1)$ & $0.65(1)$ & $0.69(1)$ \\
\hline$\psi \beta / \zeta_{\text {loc }}$ & $0.47(3)$ & $0.46(3)$ & $0.45(3)$ & $0.43(3)$ & $0.46(3)$ & $0.43(3)$ \\
$\zeta$ & \multicolumn{7}{|c}{$0.53(1)$} & & $0.68(1)$ \\
\hline \hline
\end{tabular}

TABLE II: Scaling exponents in the large- $D \xi(t)$ regime at different disorders $\Delta$, driving fields $H_{0}$ and frequencies $f$. As the creep correlation length $D \xi(t)$ grows, a significant deviation of the roughness exponent $\zeta$ from $\zeta_{l o c} \approx 2 / 3$ is observed in the right columns.

\begin{tabular}{|c|c|c|c|c|c|}
\hline$\Delta$ & 1.5 & 1.0 & 0.7 & 0.5 & 0.1 \\
\hline $1 / \psi$ & $1.84(1)$ & $1.89(2)$ & $1.82(2)$ & $1.85(3)$ & $3.07(3)$ \\
\hline$\zeta_{l o c}$ & $0.65(1)$ & $0.66(1)$ & $0.66(1)$ & $0.67(1)$ & $0.66(1)$ \\
\hline$\zeta$ & \multicolumn{3}{|c|}{$\zeta=0.68(1)$} & \multicolumn{2}{|c|}{$\zeta=0.78(1)$} \\
\hline$H_{0}$ & 0.01 & 0.05 & 0.1 & 0.2 & 0.5 \\
\hline $1 / \psi$ & $1.84(2)$ & $1.86(2)$ & $1.90(2)$ & $2.11(2)$ & $2.62(2)$ \\
\hline$\zeta_{l o c}$ & $0.65(1)$ & $0.65(1)$ & $0.66(1)$ & $0.68(1)$ & $0.66(1)$ \\
\hline$\zeta$ & \multicolumn{3}{|c|}{$\zeta=0.68(1)$} & \multicolumn{2}{|c|}{$\zeta=0.94(2)$} \\
\hline$f(\mathrm{~Hz})$ & $10^{-4}$ & $10^{-3}$ & $10^{-2}$ & $10^{-1}$ & $10^{0}$ \\
\hline $1 / \psi$ & $1.90(2)$ & $1.90(3)$ & $1.88(3)$ & $1.87(3)$ & $1.84(3)$ \\
\hline$\zeta_{l o c}$ & $0.66(1)$ & $0.66(1)$ & $0.65(1)$ & $0.65(1)$ & $0.65(1)$ \\
\hline$\zeta$ & \multicolumn{3}{|c|}{$\zeta=0.69(1)$} & \multicolumn{2}{|c|}{$\zeta=0.84(1)$} \\
\hline
\end{tabular}




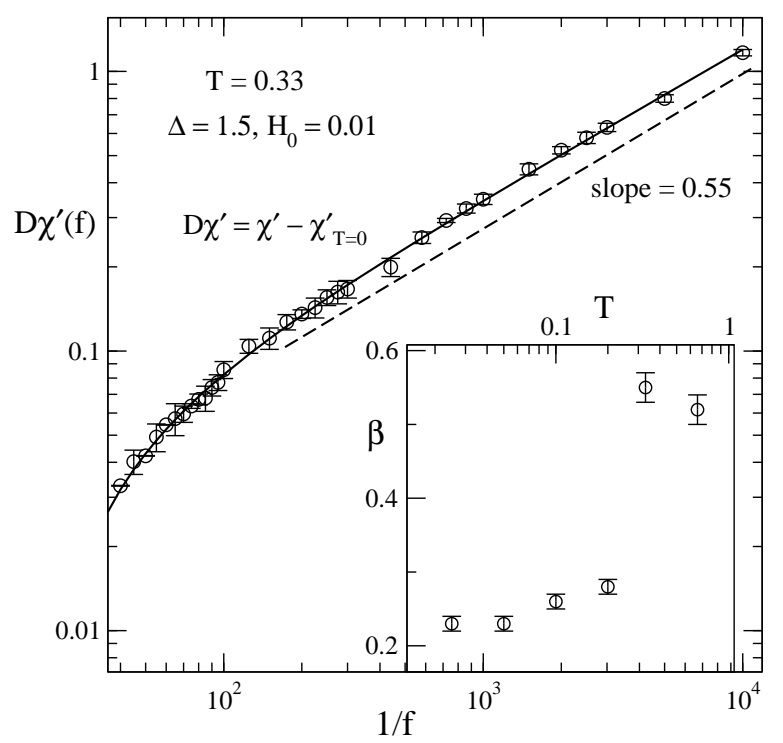

FIG. 1: The spectrum of the creep susceptibility $D \chi^{\prime}(f)$ is displayed on a log-log scale, at the temperature $T=0.33$, the strength of the disorder $\Delta=1.5$ and the driving field $H_{0}=0.01$. The dashed line represents a power-law fit, and the solid line includes the correction in the form $y=a x^{\beta}(1-c / x)$. In the inset, the creep exponent $\beta$ at different $T$ is plotted. Error bars are given when they are larger than or compatible with the symbols. 


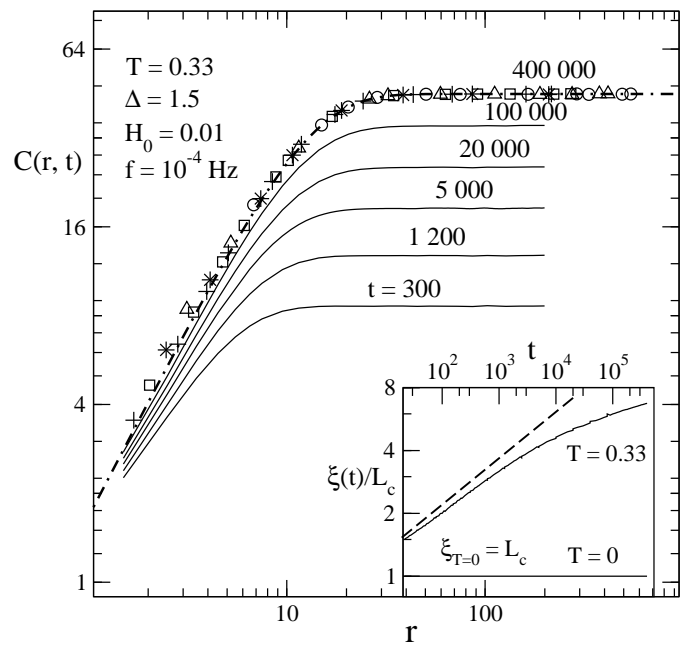

(a)

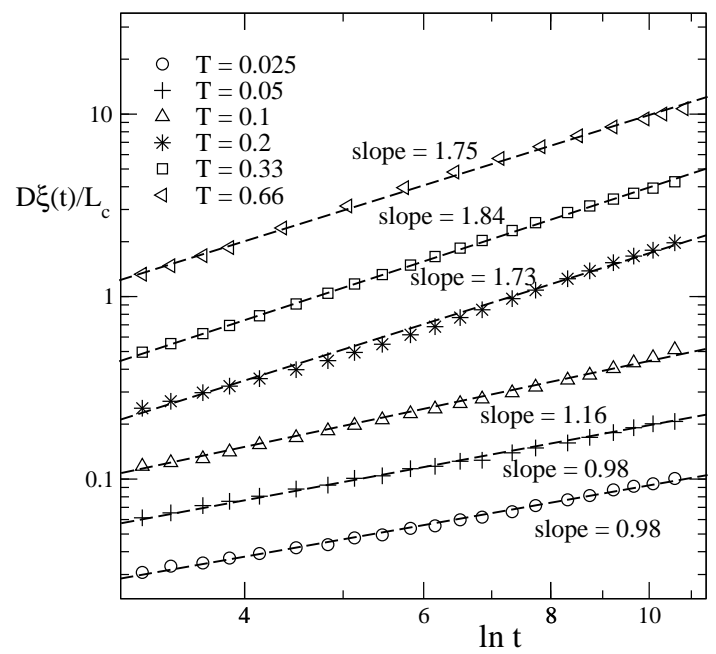

(b)

FIG. 2: (a) The correlation function $C(r, t)$ at different times $t$ are plotted with solid lines. Symbols show data collapse, and the dash-dotted line represents a fit according to Eq. (13). In the inset, the rescaled spatial correlation length $\xi(t) / L_{c}$ is plotted for $T=0$ and 0.33 on a log-log scale. (b) The creep correlation length $D \xi(t)=\xi(t)-\xi_{T=0}$ rescaled by $L_{c}$ is displayed as a function of $\ln t$ fc

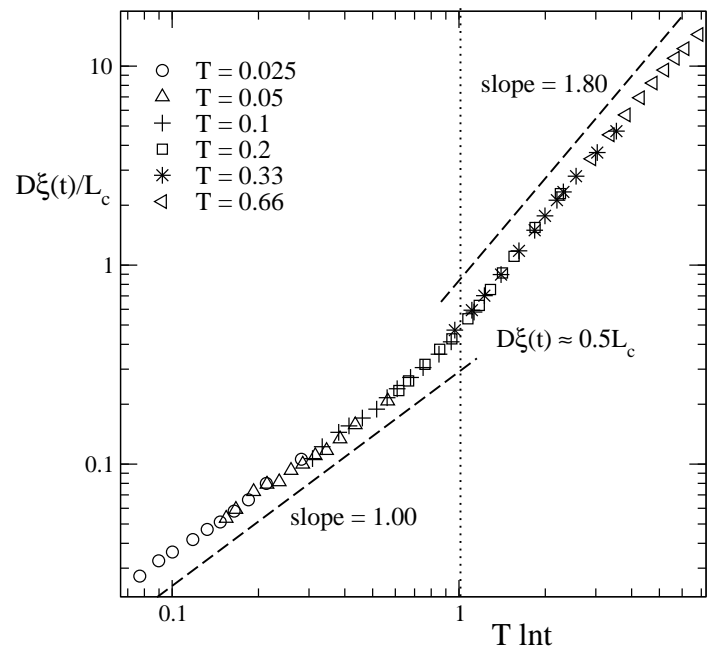

(a)

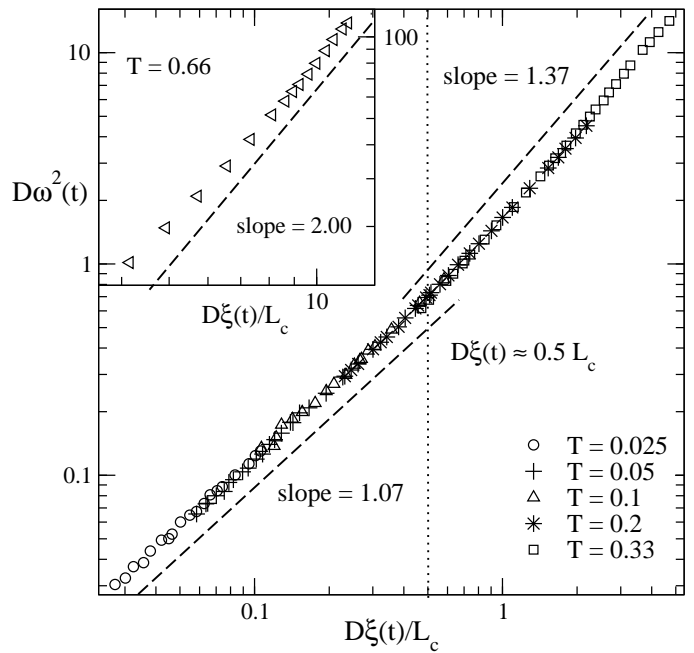

(b)

FIG. 3: (a) The creep correlation length $D \xi(t) / L_{c}$ vs. $T \ln t$ and (b) the pure roughness function $D \omega^{2}(t)$ vs. $D \xi(t) / L_{c}$ are plotted at different $T$. In the inset, the asymptotic behavior of $D \omega^{2}(t)$ is shown at $T=0.66$. In both (a) and (b), data collapse is demonstrated. Dashed lines represent power-law fits, and vertical dotted lines indicate a crossover at $D \xi(t) \approx 0.5 L_{c}$. 


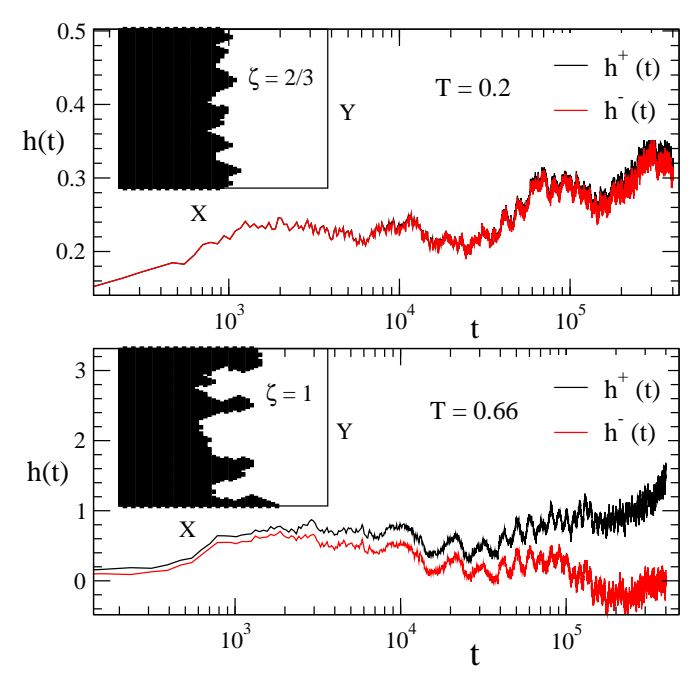

(a)

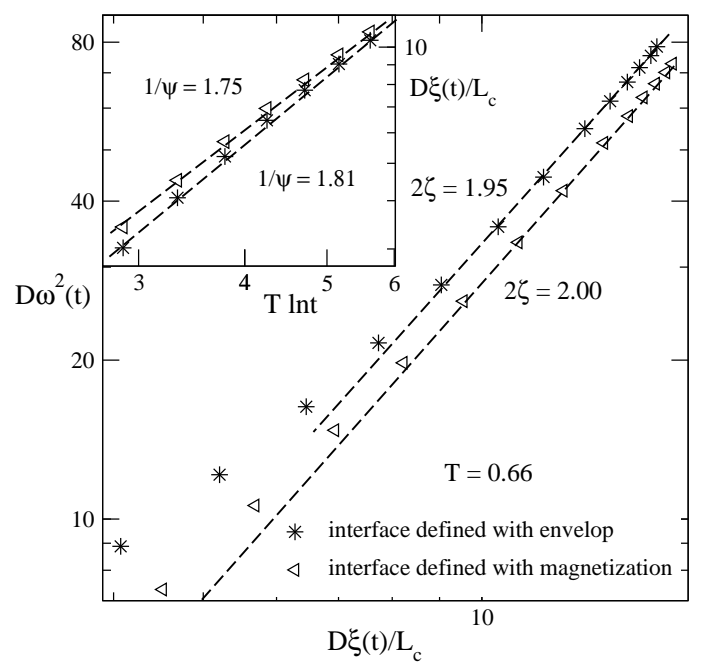

(b)

FIG. 4: (Color on-line) (a) The time evolution of the height functions $h^{+}(t)$ and $h^{-}(t)$ is plotted with black and red lines, respectively. In the insets, the snapshots of the domain interfaces at the time $t=4 \times 10^{5} \mathrm{MCS}$ are displayed for $T=0.2$ and 0.66. (b) $D \omega^{2}(t)$ and $D \xi(t) / L_{c}$ at $T=0.66$ are plotted on a log-log scale, for the domain interfaces $h(t)$ and $h^{-}(t)$ defined with the magnetization a:

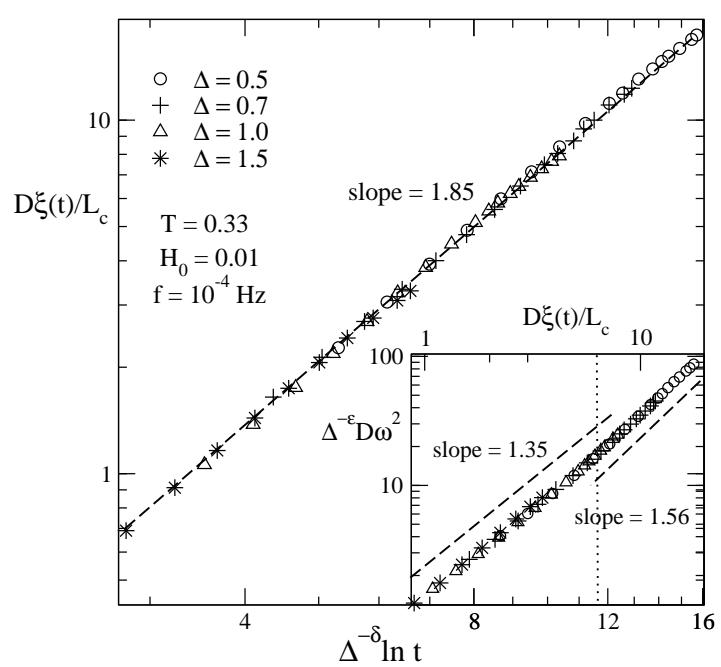

(a)

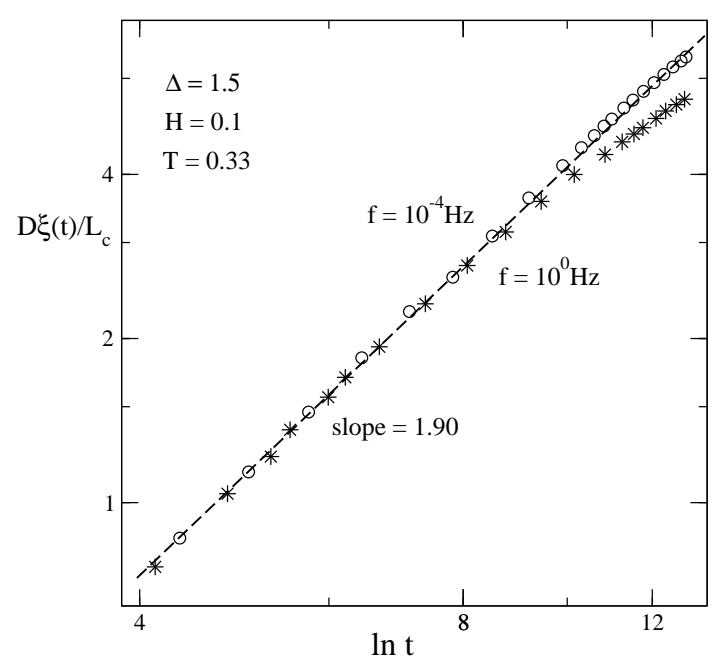

(b)

FIG. 5: (a) Taking $\delta=0.58(1)$ as input, the creep correlation length $D \xi(t) / L_{c}$ is displayed against $\Delta^{-\delta} \ln t$ for different strengthes of the disorder $\Delta$ on a $\log$-log scale. In the inset, $\Delta^{-\varepsilon} D \omega^{2}(t)$ is plotted with $\varepsilon=0.22(1)$ as input. The vertical dotted line indicates a crossover. (b) A log-log plot of $D \xi(t) / L_{c}$ vs. $\ln t$ is shown for different frequencies $f=10^{-4}$ and $10^{0} \mathrm{~Hz}$ at the driving field $H_{0}=0.1$. In both (a) and (b), dashed lines represent power-law fits. 